\title{
Expansão e mortalidade de ramos em espécies arbóreas do cerrado sensu stricto
}

\author{
DAVI RODRIGO ROSSATTO ${ }^{1,2}$ e AUGUSTO CÉSAR FRANCO ${ }^{1}$
}

(recebido: 04 de outubro de 2007; aceito: 29 de agosto de 2008)

\begin{abstract}
Shoot growth and mortality in "cerrado" sensu stricto tree species). The study of shoot growth and mortality provide essential information on tree growth dynamics. This study shows the patterns of growth and mortality in shoots of ten common tree species in a "cerrado" sensu stricto. Mean annual branch growth was variable among the species and ranged from 1 to $17 \mathrm{~cm}$ per year. Miconia pohliana Cogn. and Vochysia thyrsoidea Pohl were the ones with the highest rates of branch growth. Most shoot mortality took place during the dry season. In conclusion, shoot loss is a very common phenomenon in "cerrado" tree species and their impacts on productivity studies and growth analysis should be considered.
\end{abstract}

Key words - mortality, phenology, productivity, savanna, shoot growth

RESUMO - (Expansão e mortalidade de ramos em espécies arbóreas do cerrado sensu stricto). A abordagem de crescimento e mortalidade de ramos fornece informações essenciais a respeito da dinâmica de crescimento de espécies arbóreas. Este estudo mostrou os padrões de expansão e mortalidade de ramos em dez espécies arbóreas comuns em região de cerrado sensu stricto. O crescimento médio anual foi variável entre as espécies estando entre 1 a $17 \mathrm{~cm}$. Miconia pohliana Cogn. e Vochysia thyrsoidea Pohl foram as espécies com os maiores valores de crescimento de ramo. A mortalidade dos ramos ocorreu majoritariamente na estação seca. O estudo demonstra que a mortalidade de ramos é comum nas espécies lenhosas do cerrado e que se deve atentar para seus efeitos em análises e estudos de crescimento e de produtividade.

Palavras-chave - crescimento do ramo, fenologia, mortalidade, produtividade, savana

\section{Introdução}

Os estudos fenológicos buscam esclarecer a sazonalidade dos fenômenos biológicos, enfatizando o conjunto de características sazonais dos ambientes. A fenologia tem um papel importante em diversos processos nas comunidades vegetais, como a regeneração e reprodução de plantas e na organização temporal dos recursos, dentre outros aspectos (Lieth 1970, Morellato \& Leitão Filho 1990, 1996).

Muitos estudos têm sido realizados nos diversos ecossistemas florestais (Bencke \& Morellato 2002, San Martin-Gajardo \& Morellato 2003) e savânicos do território brasileiro (Mantovani \& Martins 1988, Batalha \& Mantovani 2000, Batalha et al. 1997, Batalha \& Martins 2004), abordando principalmente padrões reprodutivos em comunidades vegetais, a sincronia entre os indivíduos, variações populacionais e individuais,

1. Universidade de Brasília, Instituto de Ciências Biológicas, Departamento de Botânica, Laboratório de Fisiologia Vegetal, Caixa Postal 04457, 70904-970 Brasília, DF; Brasil.

2. Autor para correspondência: drrossatto@unb.br além de relações entre os fenômenos fenológicos e fatores ambientais como pluviosidade, comprimento do dia e temperatura.

Os estudos fenológicos relacionados aos padrões vegetativos em nível de indivíduo, como produção e queda de folhas e expansão de ramos, são poucos na literatura, utilizando geralmente uma abordagem qualitativa, com observações visuais de mudança no aspecto geral da copa (Gouveia \& Felfili 1998, dÉça-Neves \& Morellato 2004), do que quantitativos, como os estudos de Franco (1998), Suzuki (2003) e Damascos et al. (2005).

Um aspecto importante na avaliação dos padrões fenológicos vegetativos, pouco estudado em ecossistemas tropicais, está relacionado à expansão e mortalidade de ramos. Os estudos quantitativos a respeito da expansão e mortalidade de ramos são essenciais para o entendimento de diversos processos no ecossistema, pelos seus impactos nas taxas de crescimento, produtividade primária e ciclagem de nutrientes (Negi 2006). A mortalidade é geralmente inferida indiretamente em estudos de produção de serrapilheira de ecossistemas. A produção de serrapilheira diz respeito ao estudo da produção de folhas, ramos vegetativos e reprodutivos mortos e tem 
sido extensivamente estudada em diversos ecossistemas brasileiros (De Paula \& Lemos Filho 2000, Cianciaruso et al. 2006, Kozovits et al. 2007), sendo abordado principalmente o aspecto quantitativo de sua produção, relacionando-a aos padrões de produtividade e ciclagem de materiais dentro do ecossistema, sem separar espécies individuais.

Espécies que crescem em ambientes sazonais, como as regiões de cerrado, devem ajustar a atividade de renovação de folhas, e consequentemente de seus ramos, para maximizar a sobrevivência e a produtividade (Damascos et al. 2005). O estudo da fenologia vegetativa, abordando o processo de expansão de ramos e também de sua mortalidade, está intimamente relacionado a processos fisiológicos de crescimento, status hídrico e trocas gasosas (Reich 1995). Desta forma, o objetivo deste trabalho foi determinar o crescimento e mortalidade de ramos de 10 espécies de cerrado sensu stricto ao longo de um ano, sugerindo uma abordagem diferenciada no estudo destes padrões vegetativos.

\section{Materiais e métodos}

Este estudo foi realizado em região de cerrado na RECOR Reserva Ecológica do IBGE (1556' S e 47 $52^{\prime}$ ' W), localizada a $35 \mathrm{~km}$ do centro de Brasília-DF. A reserva apresenta um clima sazonal com uma estação seca compreendida entre os meses de maio e setembro e uma estação chuvosa, entre outubro e abril (figura 1A). Os dados climáticos foram obtidos da estação meteorológica da RECOR. Para este estudo foram utilizadas 10 espécies comumente encontradas no cerrado (Silva Junior 2005): Byrsonima crassa Nied.; Guapira noxia (Netto) Lundell; Hymenaea stigonocarpa Mart. ex Hayne; Miconia pohliana Cogn.; Myrcia tomentosa (Aubl.) DC.; Myrsine guianensis (Aubl.) Kuntze; Symplocos rhamnifolia A. DC.; Styrax ferrugineus Nees \& Mart.; Tabebuia ochracea (Cham.) Standl. e Vochysia thyrsoidea Pohl. Foram amostrados cinco indivíduos de cada espécie, selecionados em uma mesma área de cerrado sensu stricto e que apresentavam entre 6 e $8 \mathrm{~cm}$ de diâmetro a $30 \mathrm{~cm}$ do nível solo. Em cada indivíduo foram selecionados ao acaso cinco ramos terminais de tamanho semelhante, no mês de julho de 2006 (mês inicial).

O ramo foi aqui determinado como a porção definida entre a região meristemática apical e a primeira bifurcação, sendo esta determinação semelhante para todas as espécies estudadas, independentemente dos padrões de crescimento e de filotaxia destas. Todos os ramos selecionados, onde as folhas estavam inseridas, foram marcados com fios coloridos para acompanhamento de seu crescimento. Para facilitar o processo de coleta de dados, foram selecionados majoritariamente ramos que estavam nas regiões mais baixas da copa, ocorrente na periferia da copa, exposta ao sol. O tamanho inicial do ramo foi tomado, sendo colocados fios coloridos na sua porção inicial
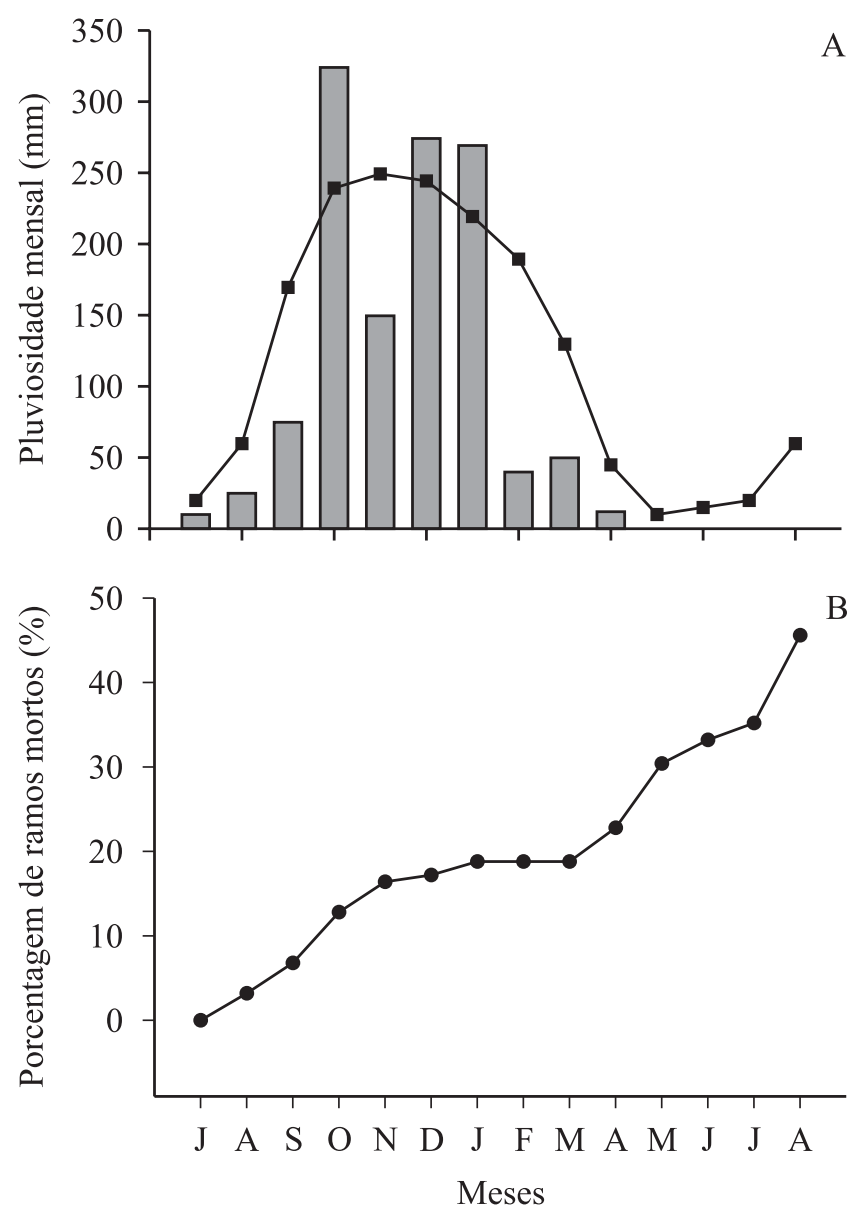

Figura 1. Valores mensais de pluviosidade (A) e porcentagem de ramos mortos (B) na comunidade de cerrado estudada. $n=25$ ramos. Normal climatológica entre 1960-1990 (-匹- ). Precipitação no período de estudo ( $\square$ ). Porcentagem de

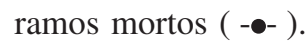

Figure 1. Monthly rainfall (A) and cumulative percentage of dead shoots (B) in a cerrado tree comunity. $n=25$ branches. Average values of monthly rainfall during the period of 1960-1990 (- - ) . Rainfall during the study period ( $\square)$. Percentage of dead branches (-๑-).

(próxima à bifurcação) e na porção terminal no mês inicial. $\mathrm{O}$ acompanhamento dos ramos, para o estudo do crescimento, foi realizado mensalmente, entre julho de 2006 e julho de 2007, com o auxílio de um paquímetro digital (Mitutoyo, resolução de $0,03 \mathrm{~mm}$ ) para tomar as medidas de comprimento.

A mortalidade dos ramos marcados foi computada mensalmente em números de ramos mortos ao longo do período que se estendeu de julho de 2006 a agosto de 2007. Considerou-se o ramo morto quando este perdia todas as folhas e secava. Calculou-se a taxa de crescimento médio mensal dos ramos, dividindo-se o crescimento médio acumulado pelos doze meses de observação. Para determinar a influência de ramos que senesceram no período, a taxa de 
crescimento dos ramos foi também calculada sem incluir os valores dos ramos que morreram no período de estudo. A idade dos ramos foi estimada utilizando-se o tamanho inicial do ramo e o crescimento médio anual dos ramos que permaneceram vivos durante os 12 meses em que foi medido o crescimento. O teste-T pareado (Zar, 1999) foi utilizado para se comparar os dados de crescimento de ramo considerando ramos mortos e ramos vivos.

\section{Resultados e discussão}

Sem considerar os ramos que morreram no período, o crescimento médio anual, para os 12 meses analisados, em comprimento dos ramos para todas as espécies foi de aproximadamente $7,42 \mathrm{~cm}$, com uma ampla faixa de variação entre 1 a $17 \mathrm{~cm}$, dependendo da espécie (tabela 1); considerando os ramos que morreram no período, o crescimento médio anual diminuiu para $5,97 \mathrm{~cm}$, com uma variação entre 1 e $16 \mathrm{~cm}$. Esta variabilidade nas taxas de crescimento anuais de ramos entre espécies lenhosas do cerrado é confirmada pela literatura: 2,5 cm para Roupala montana Aubl. (Franco 1998); 7,95 cm para Bauhinia rufa Steud.; 16,89 cm em Miconia albicans (Sw.) Steud.; e 20,89 $\mathrm{cm}$ para Leandra lacunosa Cogn. (Damascos et al. 2005). As taxas de crescimento mensais considerando todos os ramos selecionados foram bem variáveis estando entre 0,08 e $1,21 \mathrm{~cm} \mathrm{mês}^{-1}$, apresentando um intervalo maior quando se desconsideram os ramos que morreram no período (tabela 1).

A análise de crescimento dos ramos (tabela 1) foi alterada significativamente quando se considerou os ramos que morreram durante o período de avaliação do crescimento tanto para o crescimento anual (teste-T, $P=0,011)$ como para os valores de crescimento mensal (teste-T, $P=0,017$ ). O decréscimo entre as espécies foi variável, estando em uma faixa entre $2 \%$ e $30 \%$, porém para Styrax ferrugineus o decréscimo chegou a $62 \%$. Assim, quando o objetivo for analisar quantitativamente o crescimento de ramos, deve-se considerar o efeito da mortalidade durante o período analisado.

Ao se analisar a figura 1B percebe-se que, para as espécies como um todo, houve um aumento acentuado da mortalidade dos ramos durante a estação seca, entre agosto e novembro em 2006, e entre abril e agosto de 2007. Estes dados estão de acordo com estudos de produção de serrapilheira que demonstram picos de produção em julho, agosto e setembro para um cerrado em Luiz Antônio-SP (Cianciaruso et al. 2006) e entre maio e julho, do início ao meio da estação seca e, no período de transição da estação seca para a chuvosa,

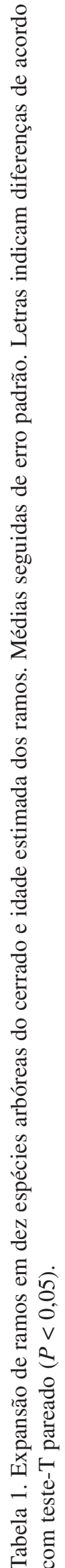

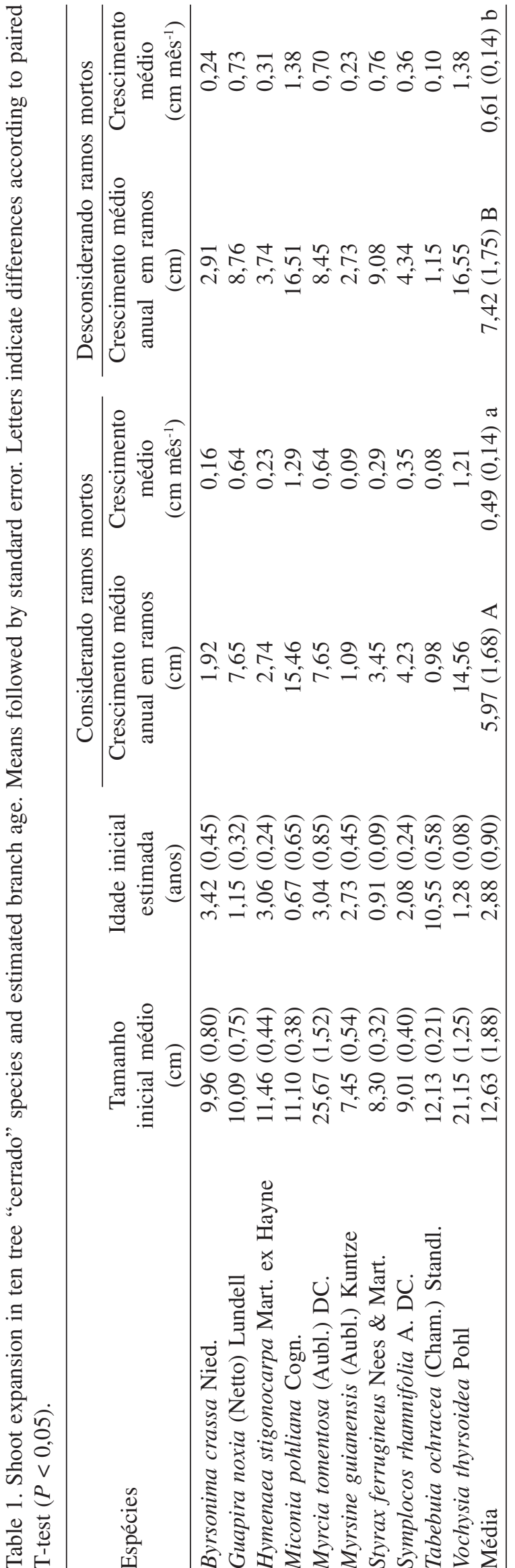


entre setembro e outubro, para espécies do cerrado do Brasil central (Kozovits et al. 2007).

Durante o período estudado todas as espécies apresentaram mortalidade de ramos (figura 2). Dos 250 ramos marcados inicialmente, 114 morreram no período de 13 meses do estudo de mortalidade, uma porcentagem de $45,6 \%$. A mortalidade foi bem variável entre as espécies, alcançando um máximo de $88 \%$ (22 ramos) em $S$. ferrugineus e um mínimo de $24 \%$ (seis ramos) para Tabebuia ochracea. No período analisado nenhum dos ramos mortos foi encontrado com sinais de predação.
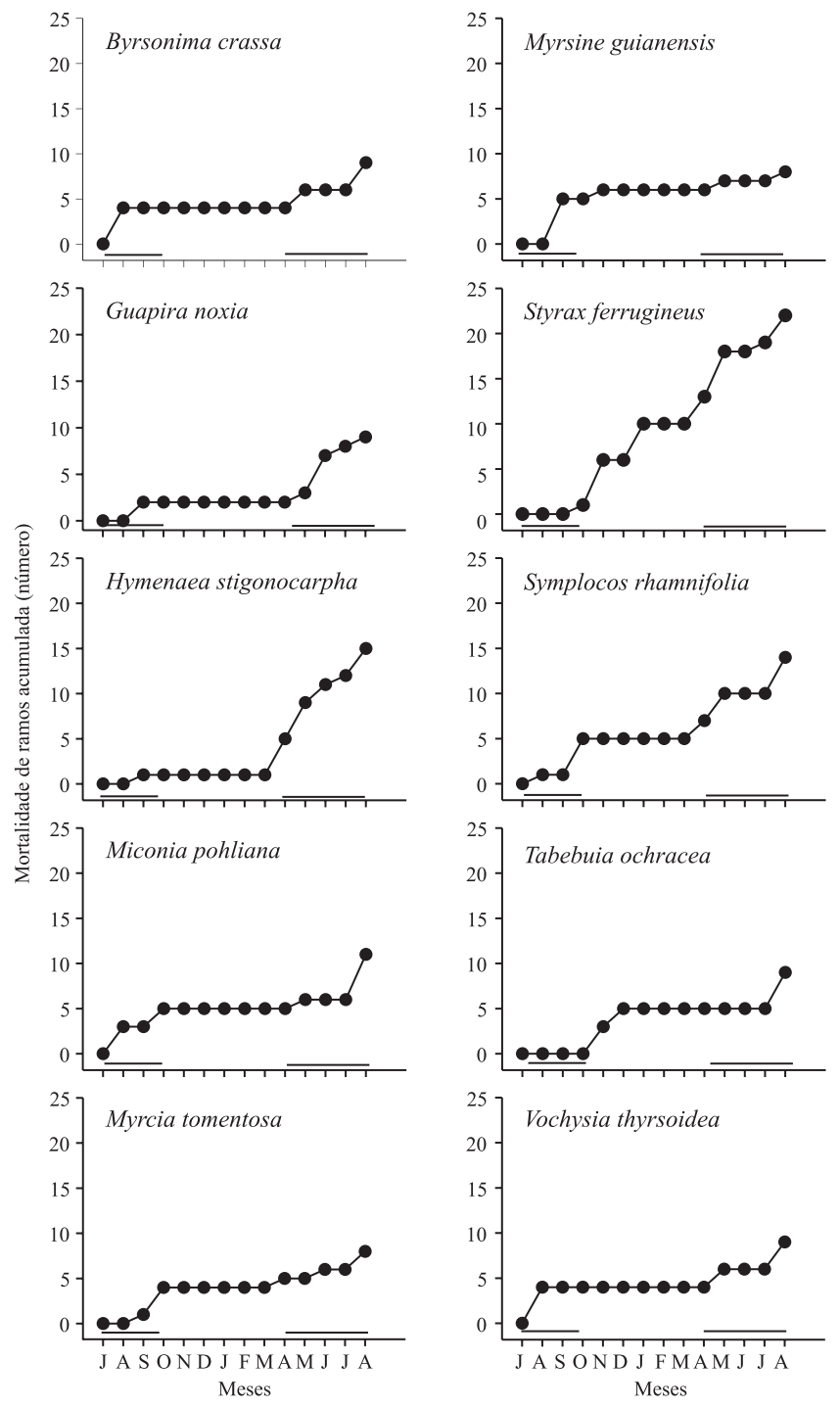

Figura 2. Mortalidade acumulada de ramos de dez espécies arbóreas do cerrado. Barras horizontais representam o período seco. $(n=25$ ramos para cada espécie).

Figure 2. Cumulative shoot mortality of ten cerrado tree species. A horizontal bar indicates the dry period. $(n=25$ branches for each species).
A mortalidade dos ramos pode estar ocorrendo nas épocas indicadas devido a causas relacionadas a processos de senescência natural (Umeki et al. 2006), principalmente pelo fato de muitos dos ramos escolhidos já terem crescido em estações anteriores, e também ao estresse causado pelo ambiente, principalmente o estresse hídrico presente durante a estação seca (Franco et al. 2005). Processos como a cavitação no xilema são bem comuns na estação seca e podem estar influenciando na mortalidade dos ramos (Rood et al. 2000). Por outro lado outros fatores podem estar ocorrendo em espécies menos sensíveis ao déficit hídrico, sendo neste caso resultado do crescimento da copa, que geralmente causa o sombreamento dos ramos mais baixos (Suzuki 2002); evidências sugerem que espécies de cerrado não são muito tolerantes ao sombreamento (Hoffmann et al. 2005).

Não se sabe ao certo a idade dos ramos escolhidos, contudo com os dados de crescimento médio anual dos ramos que permaneceram vivos e os dados de comprimento inicial, pode-se fazer uma estimativa da idade do ramo, assumindo-se uma taxa anual de crescimento constante (tabela 1). A literatura tem demonstrado que os valores de crescimento entre os diferentes anos tendem a ser bem similares, podendo variar em alguns casos, pois o crescimento é afetado pelas condições de pluviosidade e temperatura, como demonstrado por Damascos et al. (2005). Apesar de serem ramos terminais, que se desenvolveram a partir da ultima bifurcação, os resultados mostram uma grande variação entre as espécies na idade dos ramos selecionados (tabela 1).

A análise quantitativa permitiu quantificar, em média, o grau de crescimento em comprimento de ramos das espécies e desta maneira estimar a idade dos ramos; permitiu também quantificar a mortalidade dos ramos, que ocorreu predominantemente na estação seca. Esta abordagem pode auxiliar nos processos de entendimento da dinâmica na produção e significado da mortalidade de ramos, fatos que influenciam diretamente nos padrões de ciclagem e produtividade do ambiente e de balanço de carbono.

Agradecimentos - A Capes, ao CNPq e ao NSF (National Science Foundation) pelo apoio financeiro.

\section{Referências bibliográficas}

BATALHA, M.A., ARAGAKI, S. \& MANTOVANI, W. 1997. Variações fenológicas das espécies do cerrado em Emas (Pirassununga, SP) Acta Botanica Brasilica 11:61-78. 
BATALHA, M.A. \& MANTOVANI, W. 2000. Reproductive phenological patterns of cerrado plant species at the Pé-de-Gigante Reserve (Santa Rita do Passa Quatro, Sp, Brazil): a comparison between the herbaceous and woody floras. Revista Brasileira de Biologia 60:129145.

BATALHA, M.A. \& MARTINS, F.R. 2004. Reproductive phenology of the cerrado plant community in Emas National Park (Central Brazil). Australian Journal of Botany 52:149-161.

BENCKE, C.S.C. \& MORELLATO, L.P.C. 2002. Estudo comparativo da fenologia de nove espécies arbóreas em três tipos de floresta atlântica no sudeste do Brasil. Revista Brasileira de Botânica 25:237-248.

CIANCIARUSO, M.V., PIRES, J.S.R., DELITTI, W.B.C. \& DA SILVA, E.F.L.P. 2006. Produção de serrapilheira e decomposição de material foliar em um cerradão na Estação Ecológica de Jataí, município de Luiz Antônio, SP, Brasil. Acta Botanica Brasilica 20:49-59.

D'EÇA-NEVES, F.F \& MORELLATO, L.P.C. 2004. Métodos de amostragem e avaliação utilizados em estudos fenológicos de florestas tropicais. Acta Botanica Brasilica 18:99-108.

DAMASCOS, M.A., PRADO, C.H.B.A. \& RONQUIM, C.C. 2005. Bud composition, branching patterns and leaf phenology in cerrado woody species. Annals of Botany 96:1075-1084.

DE PAULA, S.A. \& LEMOS FILHO, J.P. 2000. Dinâmica de dossel em mata semidecídua no perímetro urbano de Belo Horizonte, MG. Revista Brasileira de Botânica 24:545-551.

FRANCO, A.C. 1998. Seasonal patterns of gas exchange, water relations and growth of Roupala montana, an evergreen savanna species. Plant Ecology 136:69-76.

FRANCO, A.C., BUSTAMANTE, M., CALDAS, L.S., GOLDSTEIN, G., MEINZER, F.C., KOZOVITS, A.R., RUNDEL, P. \& CORADIN, V.T.R. 2005. Leaf functional traits of Neotropical savanna trees in relation to seasonal water deficit. Trees 19:326-335.

HOFFMANN, W.A., SILVA JUNIOR, E.R., MACHADO, G.C., BUCCI, S.J., SCHOLZ, F.G., GOLDSTEIN, G. $\&$ MEINZER, F.C. 2005. Seasonal leaf dynamics across a tree density gradient in a Brazilian savanna. Oecologia 145:307-316.
KOZOVITS, A.R., BUSTAMANTE, M.M.C., FAROFALO, C.R., BUCCI, S., FRANCO, A.C., GOLDSTEIN, G. \& MEINZER, F.C. 2007. Nutrient resorption and patterns of litter production and decomposition in a Neotropical savanna. Functional Ecology 21:1034-1043.

LIETH, H. 1970. Phenology in productivity studies. In Analysis of temperate forest ecosystems (D.E. Reichle, ed.). Springer-Verlag, Berlim.

NEGI, G.C.S. 2006. Leaf and bud demography and shoot growth in evergreen and deciduous trees of central Himalaya, India. Trees 20:416-429.

MANTOVANI, W. \& MARTINS, F.R. 1988. Variações fenológicas das espécies do cerrado da Reserva Biológica de Moji-Guaçu, Estado de São Paulo. Revista Brasileira de Botânica 11:101-112.

MORELLATO, L.P.C. \& LEITÃO-FILHO, H.F. 1990. Estratégias fenológicas de espécies arbóreas em floresta mesófila na Serra do Japi, Jundiaí-SP. Revista Brasileira de Biologia 50:163-173.

MORELLATO, L.P.C. \& LEITÃO-FILHO, H.F. 1996. Reproductive phenology of climbers in a Southeastern Brazilian Forest. Biotropica 28:180-191.

REICH, P.B. 1995. Phenology of tropical forests: patterns, causes and consequences. Canadian Journal of Botany 73:164-174.

ROOD, S.B., PATIÑO, S., COOMBS, K. \& TYREE, M.T. 2000. Branch sacrifice: cavitation-associated drought adaptation of riparian cottonwoods. Trees 14:248-257.

SAN MARTIN-GAJARDO, I. \& MORELLATO, L.P.C. 2003. Fenologia de Rubiaceae do sub-bosque em floresta Atlântica no sudeste do Brasil. Revista Brasileira de Botânica 26:299-309.

SILVA JUNIOR, M.C. 2005. 100 Árvores do cerrado: guia de campo. Rede de Sementes do Cerrado, Brasília.

SUZUKI, A. 2002. Influence of shoot architectural position on shoot growth and branching patterns in Cleyera japonica. Tree Physiology 22:885-890.

SUZUKI, A.A. 2003. Shoot growth patterns in saplings of Cleyera japonica in relation to light and architectural position. Tree Physiology 23:67-71.

UMEKI, K., SEINO, T., LIM, E. \& HONJO, T. 2006. Patterns of shoot mortality in Betula platyphylla in northern Japan. Tree Physiology 26:623-632.

ZAR, J.H. 1999. Biostatistical analysis. Prentice Hall, New Jersey. 